\title{
ALMOST SURE STABILITY OF PARTIAL SUMS OF UNIFORMLY BOUNDED RANDOM VARIABLES
}

\author{
THEODORE P. HILL
}

\begin{abstract}
Suppose $a_{1}, a_{2} \ldots$ is a sequence of real numbers with $a_{n} \rightarrow \infty$. If $\lim \sup \left(X_{1}+\cdots+X_{n}\right) / a_{n}=\alpha$ a.s. for every sequence of independent nonnegative uniformly bounded random variables $X_{1}, X_{2}, \ldots$ satisfying some hypothesis condition $\mathrm{A}$, then for every (arbitrarily-dependent) sequence of nonnegative uniformly bounded random variables $Y_{1}, Y_{2}, \ldots, \lim \sup \left(Y_{1}+\cdots+Y_{n}\right) / a_{n}=\alpha$ a.s. on the set where the conditional distributions (given the past) satisfy precisely the same condition A. If, in addition, $\Sigma^{\infty} a_{n}^{-2}<\infty$, then the assumption of nonnegativity may be dropped.
\end{abstract}

1. Introduction. The purpose of this note is to provide partial answers to the question of when the following statement $(\mathrm{S} 1)$ is true in the case where "property B" is a property reflecting the limiting behavior of the stabilized (or normalized) partial sums.

If every sequence of independent random variables having property A has property B almost surely, then every sequence of random variables has property B almost surely on the set where the conditional distributions (given the past) have property A.

Of course ( $\mathrm{S} 1)$ is not true for all choices of properties $\mathrm{A}$ and $\mathrm{B}$, but it is sometimes true in rather general contexts. In [4], for example, it was shown that ( $\mathrm{S} 1)$ is true whenever "property B" is "the partial sums converge", regardless of property A. In the present note, a partial analysis of ( $\mathrm{S} 1)$ is given for conclusions concerning the limiting behavior, not of the partial sums $S_{n}$, but of the stabilized partial sums $S_{n} / a_{n}$ for suitable normalizing constants $\left\{a_{n}\right\}$.

As in [4], $\mathcal{Y}=\left(Y_{1}, Y_{2}, \ldots\right)$ is a sequence of random variables on a probability triple $(\Omega, \mathfrak{A}, P), S_{n}=Y_{1}+\cdots+Y_{n}$, and $\mathscr{F}_{n}$ is the sigma field generated by $Y_{1}, \ldots, Y_{n}$. Let $\pi_{n}(\cdot, \cdot)$ be a regular conditional distribution for $Y_{n}$ given $\widetilde{F}_{n-1}$, and let $\Pi=$ $\left(\pi_{1}, \pi_{2}, \ldots\right)$. Let $\mathscr{B}$ denote the Borel $\sigma$-field on $\mathbf{R}$, and $\Re^{\infty}$ the product $\sigma$-field on $\mathbf{R}^{\infty}$; let $\mathscr{P}(\mathbf{R})$ denote the space of probability measures on $(\mathbf{R}, \mathscr{B})$, and let $\mathcal{C}=\mathscr{P}(\mathbf{R}) \times$ $\mathscr{P}(\mathbf{R}) \times \cdots$. (As mentioned in [4], it might help the reader to think of $\mathcal{Y}$ as a random

Received by the editors June 10, 1982 and, in revised form, April 4, 1983.

1980 Mathematics Subject Classification. Primary 60F 15; Secondary 60G45.

Key words and phrases. Almost-sure stability of partial sums, martingale strong law of large numbers, conditional generalizations of strong laws.

'Partially supported by a NATO Postdoctoral Fellowship. 
element of $\mathbf{R}^{\infty}$, and of $\Pi$ as a random element of $\mathcal{C}$.) $\mathcal{L}(X)$ is the distribution of the random variable $X$.

Let $B \in G B^{\infty}$. In this notation, the formalization of statement (S1) is the following statement (S2) of [4].

If $A \subset \varrho$ is such that $\left(X_{1}, X_{2}, \ldots\right) \in B$ a.s. whenever $X_{1}$, $X_{2}, \ldots$ are independent and $\left(\mathcal{L}\left(X_{1}\right), \mathcal{L}\left(X_{2}\right), \ldots\right) \in A$, then for arbitrary $\mathcal{O}, \mathcal{Y} \in B$ a.s. on the set where $\Pi \in A$.

Definition 1. $\mathscr{K}=\left\{B \in \mathscr{B}^{\infty}:(\mathrm{S} 2)\right.$ is true for all $\left.A \subset \mathcal{C}\right\}$.

Throughout this note, $a_{1}, a_{2}, \ldots$ and $b_{1}, b_{2}, \ldots$ are sequences of real numbers with $a_{n} \rightarrow \infty$ and $\Sigma^{\infty} b_{n}^{-2}<\infty$, and $\vec{r}=\left(r_{1}, r_{2}, \ldots\right)$. The main positive result in this paper is the following theorem.

THEOREM 1. For all $M>0$, all $-\infty<a<b<\infty$, and all $D \subset[-\infty, \infty]$,

(i) $B=B_{0}^{M}=\left\{\vec{r} \in \mathbf{R}^{\infty}: 0 \leqslant r_{i} \leqslant M\right.$ for all $i$, and $\lim _{\sup _{n \rightarrow \infty}}\left(r_{1}+\cdots+r_{n}\right) / a_{n} \in$ $D\} \in \mathcal{K}$;

and

(ii) $B=B_{a}^{h}=\left\{\vec{r} \in \mathbf{R}^{\infty}: a \leqslant r_{i} \leqslant b\right.$ for all $i$, and $\lim _{\sup _{n \rightarrow \infty}}\left(r_{1}+\cdots+r_{n}\right) / b_{n} \in$ D\} $\in \mathcal{K}$.

Moreover, the analogs of (i) and (ii) with lim sup replaced by lim inf or lim also hold.

In contrast to the conclusions of Theorem 1, examples will be given in $\$ 4$ to show that

$$
\begin{aligned}
& B=\left\{\vec{r} \in \mathbf{R}^{\infty}:-1 \leqslant r_{i} \leqslant 1 \text { for all } i,\right. \text { and } \\
&\left.\quad \limsup _{n \rightarrow \infty}\left(r_{1}+\cdots+r_{n}\right) /(n \log \log (n / 2))^{1 / 2}=1\right\} \notin \mathscr{K} ;
\end{aligned}
$$

and that

$$
B=\left\{\vec{r} \in \mathbf{R}^{\infty}: \lim _{n \rightarrow \infty}\left(r_{1}+\cdots+r_{n}\right) / n \neq 0\right\} \notin \mathscr{K} .
$$

2. Applications of Theorem 1. Theorem 1(i) essentially says that any theorem for nonnegative uniformly bounded independent random variables which concludes "lim $\sup _{n \rightarrow \infty} S_{n} / a_{n} \in D$ a.s." may immediately be generalized into a conditional version of that result. For example, suppose one just learned the following fact.

If $X_{1}, X_{2}, \ldots$ are independent random variables taking values in $[0,1]$, and if $P\left(X_{n} \leqslant x\right) \geqslant 1-x^{n-1}$ for all $x \in[0,1]$ and all $n$, then

$$
\lim \sup \left(X_{1}+\cdots+X_{n}\right) / \log n \leqslant 1 \text { a.s. }
$$

Applying Theorem 1(i) with $a_{n}=\log n, M=1$, and $D=[0,1]$ allows the generalization of (3) given by (4).

If $Y_{1}, Y_{2}, \ldots$ are (arbitrarily-dependent) random variables taking values in $[0,1]$, then $\lim \sup _{n \rightarrow \infty} S_{n} / \log n \leqslant 1$ a.s. on the set where $P\left(Y_{n} \leqslant x \mid \mathscr{F}_{n-1}\right) \geqslant 1-x^{n-1}$ for all $x \in[0,1]$ and all $n$. 
Clearly it is not difficult to prove (4) using the conditional versions of the arguments used to establish (3), as is often the case; the point of Theorem 1 is that this is not necessary.

As an application of Theorem 1(ii), one may prove Levy's martingale strong law of large numbers $[5, \S 69$, p. 250$]$ by proving the special case of independence, and then applying Theorem l(ii) with $b_{n}=n$ and $D=\{0\}$. For a stronger similar result, consider first the following theorem (5).

[6, Theorem 2.8.1]. Let $X_{1}, X_{2}, \ldots$ be a sequence of independent, mean zero uniformly bounded random variables. Then $\left(X_{1}+\cdots+X_{n}\right) / n^{1 / 2+\varepsilon} \rightarrow 0$ a.s. for each $\varepsilon>0$.

Applying Theorem 1(ii) one immediately has the following martingale generalization of (5).

Let $S_{1}, S_{2}, \ldots$ be a martingale with uniformly bounded increments. Then $S_{n} / n^{1 / 2+\varepsilon} \rightarrow 0$ a.s. for each $\varepsilon>0$.

(Stronger results than (6) are known; see, for example, Chow's result [2 or 7, Theorem 3.3.1].) It should be noted that the classical formulations of most laws of the iterated logarithm do not fit the framework of Theorem 1; they usually involve some form of centering which violates the nonnegativity assumption of (i), and clearly $b_{n}=(n \log \log n)^{1 / 2}$ does not satisfy the $\Sigma^{\infty} b_{n}^{-2}<\infty$ hypothesis of (ii).

Although the above applications all involve conditional moment hypotheses, the "hypothesis"set $A$ in (S2) need neither be measurable nor involve (conditional) moments. (For a similar nonmoment application, the reader may see [4, Theorem 2].) It should also be remarked that technically speaking the set $D$ in Theorem 1 may also be nonmeasurable, although in most applications, $D$ will simply be a point or interval.

3. Proof of Theorem 1. The argument below closely parallels that in the proof of [4, Theorem 1]; the crucial difference being the use of Lemma 2 in place of the three-series and Borel-Cantelli arguments of [4]. Without loss of generality, assume $(\Omega, \mathfrak{A}, P)$ is complete.

LEMMA 1 [4, LeMmA 1]. (S2) $\Leftrightarrow$ (S3).

$$
\begin{aligned}
& P\left(\left\{\omega: P_{\Pi(\omega)}(B)=1\right\} \cap \mathcal{Y} \notin B\right)=0 \text { for all } \mathcal{Y} \text {, where } P_{\Pi(\omega)} \text { is } \\
& \text { the product measure } \pi_{1}(\omega) \times \pi_{2}(\omega) \times \cdots \text { on }\left(\mathbf{R}^{\infty}, \mathscr{B}^{\infty}\right) .
\end{aligned}
$$

REMARKS. In Lemma 1, the "hypothesis" set A of (S2) has in effect been replaced by the set representing all possible sufficient conditions for the conclusion " $\left(X_{1}, X_{2}, \ldots\right) \in B$ " to hold; one direction of the equivalence uses the completeness of the measure space to guarantee all subsets of null sets are measurable. In terms of the class $\mathscr{K}$, Lemma 1 says

$$
\mathscr{K}=\left\{B \in \mathscr{B}^{\infty}: P\left(\left\{\omega: P_{\Pi(\omega)}(B)=1\right\} \cap \mathcal{Y} \notin B\right)=0 \text { for all } \mathcal{Y}\right\} .
$$

LEMMA 2. Let $Y_{1}, Y_{2}, \ldots$ be a sequence of uniformly bounded (arbitrarily-dependent) random variables, and let $s_{n}=\sum_{1}^{n} E\left(Y_{j} \mid \mathscr{F}_{j-1}\right)$. Then

(i) $\lim \sup _{n \rightarrow \infty} S_{n} / b_{n}=\lim \sup _{n \rightarrow \infty} s_{n} / b_{n}$ a.s.; and

(ii) if $Y_{i} \geqslant 0$ for all $i$, then $\lim \sup _{n \rightarrow \infty} S_{n} / a_{n}=\limsup _{n \rightarrow \infty} s_{n} / a_{n}$ a.s. 
Proof. (i) follows from a martingale result of Chow [2 or 7, Theorem 3.3.1].

(ii) follows easily (since $a_{n} \rightarrow \infty$ ) from a generalization of a result of Dubins and Freedman [3] which can be found in Brown [1], namely:

If $Y_{1}, Y_{2}, \ldots$ are nonnegative uniformly bounded random variables, then $\lim _{n \rightarrow \infty} S_{n} / s_{n}<\infty$ a.s., and $=1$ almost surely on the set $s_{n} \rightarrow \infty$.

The following example shows that the "uniform boundedness" assumption in Lemma 2 may not be replaced by "tightness".

EXAMPLE 1. Let $Y_{1}, Y_{2}, \ldots$ be independent with

$$
P\left(Y_{n}=n^{2}\right)=n^{-2}=1-P\left(Y_{n}=0\right) .
$$

Then $s_{n} / n \equiv 1$, but $\lim \sup _{n \rightarrow \infty} S_{n} / n=0$ a.s. by the Borel-Cantelli Lemma.

Proof of Theorem 1. The argument for (i) only will be given; that for (ii) and lim inf or lim are similar.

Fix $M>0$ and $D \subset[-\infty, \infty]$. By (7), it suffices to show that $B$ satisfies (S3), where $B=B_{0}^{M}=\left\{\vec{r} \in \mathbf{R}^{\infty}: 0 \leqslant r_{i} \leqslant M\right.$ for all $i$, and $\lim \sup _{n \rightarrow \infty}\left(r_{1}+\cdots+r_{n}\right) / a_{n}$ $\in D\}$. Fix $\mathcal{Y}=\left(Y_{1}, Y_{2}, \ldots\right)$.

Let $(\hat{\Omega}, \hat{\mathfrak{A}}, \hat{P})$ be a copy of $(\Omega, \mathfrak{A}, P)$, and (enlarging this new space if necessary) for each $\omega \in \Omega$, let $Z_{1}(\omega), Z_{2}(\omega), \ldots$ be a sequence of independent random variables on $(\hat{\Omega}, \hat{\mathfrak{A}}, \hat{P})$ with $\mathcal{L}\left(Z_{n}(\omega)\right)=\pi_{n}(\omega)$. Let $E=\left\{\omega \in \Omega\right.$ : support of $\pi_{n}(\omega) \subset[0, M]$ for all $n\}$, and observe that, without loss of generality, it may be assumed that $E=\left\{\omega \in \Omega: 0 \leqslant Z_{n}(\omega) \leqslant M\right.$ everywhere (in $\hat{\Omega}$ ) for all $\left.n \geqslant 1\right\}$. Next, calculate

$$
\begin{aligned}
& P\left(\left\{\omega: P_{\Pi(\omega)}(B)=1\right\} \cap \mathcal{Y} \notin B\right) \\
& =P\left(E \cap\left\{\omega: P_{\Pi(\omega)}(B)=1\right\} \cap \mathcal{Y} \notin B\right) \\
& =P\left(\left\{\omega: 0 \leqslant Z_{i}(\omega) \leqslant M \text { for all } i\right.\right. \text { and } \\
& \left.\left.\limsup _{n \rightarrow \infty}\left(Z_{1}(\omega)+\cdots+Z_{n}(\omega)\right) / a_{n} \in D \text { a.s. }(\text { in }(\hat{\Omega}, \hat{\mathfrak{A}}, \hat{P}))\right\} \cap \mathcal{Y} \notin B\right) \\
& =P\left(\left\{\omega: 0 \leqslant Z_{i}(\omega) \leqslant M \text { for all } i\right.\right. \text { and } \\
& \left.\left.\limsup _{n \rightarrow \infty}\left(\hat{E} Z_{1}(\omega)+\cdots+\hat{E} Z_{n}(\omega)\right) / a_{n} \in D\right\} \cap \mathcal{Y} \notin B\right) \\
& =P\left(E \cap \left\{\omega: \limsup _{n \rightarrow \infty}\left(E Y_{1}+E\left(Y_{2} \mid \mathscr{F}_{1}\right)(\omega)\right.\right.\right. \\
& \left.\left.\left.+\cdots+E\left(Y_{n} \mid \mathscr{F}_{n-1}\right)(\omega)\right) / a_{n} \in D\right\} \cap \mathcal{Y} \notin B\right)
\end{aligned}
$$

$=0$,

where the first equality in (9) follows by the definitions of $E$ and $B$; the second by the definitions of $Z_{n}(\omega)$ and $B$; the third by Lemma 2(ii) (recall that $Z_{1}(\omega)$, $Z_{2}(\omega), \ldots$ are independent in $(\hat{\Omega}, \hat{\mathfrak{A}}, \hat{P})$ for each $\left.\omega\right)$; the fourth by the definition of $Z_{n}(\omega)$ and $\pi_{n}$; and the last by Lemma 2(ii) again and the definitions of $B$ and $E$. 
4. Examples establishing (1) and (2).

EXAMPLE 2 [4]. Let $Y_{n}=S_{n}-S_{n-1}$, where $S_{1}, S_{2}, \ldots$ are iid, $P\left(S_{n}=0\right)$ $=P\left(S_{n}=1\right)=\frac{1}{2}$. Then for $a_{n}=(n \log \log (n / 2))^{1 / 2}$ and $D=\{1\}, \lim \sup _{n \rightarrow \infty} S_{n} / a_{n}$ $=0 \notin D$ a.s., but $P_{\mathrm{II}(\omega)}(B)=1$ a.s. for

$$
B=\left\{\vec{r} \in \mathbf{R}^{\infty}:-1 \leqslant r_{i} \leqslant 1 \text { for all } i, \text { and } \limsup _{n \rightarrow \infty}\left(r_{1}+\cdots+r_{n}\right) / a_{n}=1\right\} .
$$

Via (7), this proves (1).

EXAmple 3. Let $S_{1}, S_{2}, \ldots$ be independent with $P\left(S_{n}=\sqrt{n}\right)=P\left(S_{n}=-\sqrt{n}\right)=\frac{1}{2}$ and let $Y_{n}=S_{n}-S_{n-1}\left(Y_{1}=S_{1}\right)$. From the definition of $S_{n}$, one has that $\lim S_{n} / n$ $=0$ a.s. There are only two possible conditional laws for $\pi_{n}$, namely

$$
\pi_{n}^{(+)}=(\delta(\sqrt{n}+\sqrt{n-1})+\delta(-\sqrt{n}+\sqrt{n-1})) / 2
$$

and

$$
\pi_{n}^{(-)}=(\delta(\sqrt{n}-\sqrt{n-1})+\delta(-\sqrt{n}-\sqrt{n-1})) / 2 .
$$

Construct $\left\{Z_{n}\right\}$ as in the proof of Theorem 1. Then the unconditional distribution of $\left\{Z_{n}\right\}$ is independent with law

$$
\begin{aligned}
P\left(Z_{n}=\sqrt{n}+\sqrt{n-1}\right) & =P\left(Z_{n}=\sqrt{n}-\sqrt{n-1}\right)=P\left(Z_{n}=-\sqrt{n}+\sqrt{n-1}\right) \\
& =P\left(Z_{n}=-\sqrt{n}-\sqrt{n-1}\right)=\frac{1}{4} .
\end{aligned}
$$

It will now be shown that

$$
\left(Z_{1}+\cdots+Z_{n}\right) / n \nrightarrow 0 \text { a.s. }
$$

To see (10), consider the following theorem of Revesz [6, p. 65 or 7, p. 167]:

$$
\text { If } Z_{1}, Z_{2}, \ldots \text { are independent with }\left|Z_{n}\right| \leqslant n \text { a.s. and } E\left(Z_{n}\right)=0
$$

$$
\begin{aligned}
& \text { for all } n \geqslant 1, \text { then }\left(Z_{1}+\cdots+Z_{n}\right) / n \rightarrow 0 \quad \text { a.s. implies } \\
& \lim _{n \rightarrow \infty}\left[\sum^{n} E\left(Z_{i}^{2} / i^{2}\right)\right] / \log n=0 .
\end{aligned}
$$

Since $E\left(Z_{n}^{2}\right)=2 n-1$ for all $n$, then $\lim _{n \rightarrow \infty} \sum^{n} E\left(Z_{i}^{2} / i^{2}\right) / \log n=0$ which by (11) implies (10). Since $\lim _{n \rightarrow \infty} S_{n} / n=0$ a.s., this establishes (2). (Informally, (2) says that " $S_{n} / n$ does not converge to zero" is not a "property B" for which (S1) holds for every property A.)

ACKNOWLEDGements. The author is grateful to Robert Kertz for several useful conversations, to Thomas Kurtz and the referee for several suggestions, and to the Department of Mathematics at the University of Leiden for its hospitality and technical assistance during the academic year 1982-83.

\section{REFERENCES}

1. B. M. Brown, A conditional setting for some theorems associated with the strong law, Z. Wahrsch. Verw. Gebiete 19 (1971), 274-280.

2. Y. S. Chow, Local convergence of martingales and the laws of large numbers, Ann. Math. Statist. 36 (1965), 800-807. 
3. L. Dubins and D. Freedman, A sharper form of the Borel-Cantelli lemma and the strong law, Ann. Math. Statist. 36 (1965), 552-558.

4. T. P. Hill, Conditional generalizations of strong laws which conclude the partial sums converge almost surely, Ann. Probab. 10 (1982), 828-830.

5. P. Levy, Théorie de l'addition des variables aléatoires, Gauthier-Villars, Paris, 1937.

6. P. Revesz, The laws of large numbers, Academic Press, New York, 1968.

7. W. F. Stout, Almost sure convergence, Academic Press, New York, 1974.

Department of Mathematics, University of Leiden, Leiden, The Netherlands

Current address: School of Mathematics, Georgia Institute of Technology, Atlanta, Georgia 30332 